\title{
Solar particle event detected by ALTEA on board the International Space Station
}

\section{The March 7th, 2012 X5.4 flare}

\author{
Luca Di Fino ${ }^{1,2, *}$, Veronica Zaconte ${ }^{1,2}$, Marco Stangalini ${ }^{3}$, Roberta Sparvoli ${ }^{1,2}$, Piergiorgio Picozza ${ }^{1,2}$, \\ Roberto Piazzesi ${ }^{1}$, Livio Narici ${ }^{1,2}$, Marianna Larosa ${ }^{1,2}$, Dario Del Moro ${ }^{1}$, Marco Casolino ${ }^{2,4}$, \\ Francesco Berrilli ${ }^{1}$, and Stefano Scardigli ${ }^{1}$ \\ 1 Department of Physics, University of Roma Tor Vergata, Via Ricerca Scientifica 1, 00133 Rome, Italy \\ *Corresponding author: e-mail: luca.difino@roma2.infn.it \\ 2 INFN-National Institute for Nuclear Physics, Structure of Rome Tor Vergata Group, Italy \\ 3 INAF-Osservatorio Astronomico di Roma, 00040 Monte Porzio Catone (RM), Italy \\ 4 RIKEN, Hirosawa, Wako, Saitama 351-0198, Japan
}

Received 31 December 2013 / Accepted 3 May 2014

\begin{abstract}
Context. Solar activity poses substantial risk for astronauts of the International Space Station (ISS) both on board and during extravehicular activity. An accurate assessment of the charged radiation flux in space habitats is necessary to determine the risk and the specific type of radiation exposure of ISS crew members, and to develop ways to protect future crews for planetary missions, even in case of high solar activity.

Aims. To reduce the present-day uncertainties about the nature and magnitude of the particle fluxes in space habitats during a solar event, it is fundamental to measure those fluxes in situ.

Methods. The ALTEA (Anomalous Long Term Effects on Astronauts) experiment on board the ISS is an active detector composed of six silicon telescopes and is able to follow the dynamics of the radiation flux. During its operation in 2012 a number of flux peaks were detected in correspondence with solar events.

Results. We present in this work an analysis of the ALTEA data measured during the March 7th, 2012 solar event, produced by NOAA AR11429.

Conclusions. During this event, the flux was enhanced tenfold with respect to "quiet Sun" conditions, producing strong dose increases at high geomagnetic latitudes.
\end{abstract}

Key words. space weather - solar activity - flare - Coronal Mass Ejection (CME)

\section{Introduction}

Radiation exposure is one of the principal causes of danger to the safety of astronauts, principally during space missions outside low Earth orbits (LEO) or during extravehicular activities (EVAs). Under these conditions, space crew exposure rates will go beyond levels usually received by on-ground radiation operators. A detailed knowledge of the radiation environment and its connection with natural sources is essential for an appropriate risk assessment and mitigation (Turner 1996; Schimmerling et al. 2003; Durante \& Cucinotta 2011) and to forecast potential hazardous events (Lapenta et al. 2013; Tsagouri et al. 2013).

A serious radiation risk for astronauts arises from highenergy charged particles. This space radiation primarily consists of Hydrogen nuclei (protons), Helium nuclei ( $\alpha$ particles) and about $1 \%$ of heavier elements. Various natural sources are able to originate these particles, among them galactic cosmic rays (GCR), solar energetic particles (SEP) and, if travelling in low Earth orbit (LEO), trapped radiation in the Van Allen belts. A different distribution of particle energies and fluence characterize each source. Nevertheless, all these sources are modulated by both solar impulsive activity and the 11-y cycle (Potgieter 2013).
More in detail, GCR originate from outside the solar system and are characterized by very large kinetic energies (up to several thousand $\mathrm{GeV}$ nucleon ${ }^{-1}$ ). These particles are distributed isotropically and found in relatively low fluence in respect to SEPs and trapped particle components. GCR integral intensity is reduced during the solar maximum activity due to the shielding of Earth by the enhanced interplanetary magnetic field produced by the Sun. Particularly, during the solar minimum the GCR dose rate in free space is about 2.5 times higher than at solar maximum as measured by the Space Radiation Analysis Group, Johnson Space Center ${ }^{1}$.

Solar Particle Events (SPE) consist of the ejection of energetic particles from the solar corona during explosive processes such as flares and Coronal Mass Ejections (CME). During such processes, triggered by photospheric flows and magnetic (Shibata \& Magara 2011), high energy photons ( $\gamma$ - or X-rays) and energetic electrons, protons, $\alpha$ and heavier particles are injected into interplanetary space. The frequency and intensity of flares and CMEs increase during periods of maximum solar activity. Severe space weather conditions for astronauts are generated especially by intense flares occurring at precise solar

\footnotetext{
${ }^{1}$ http://srag-nt.jsc.nasa.gov/Publications/Publications.cfm.
} 


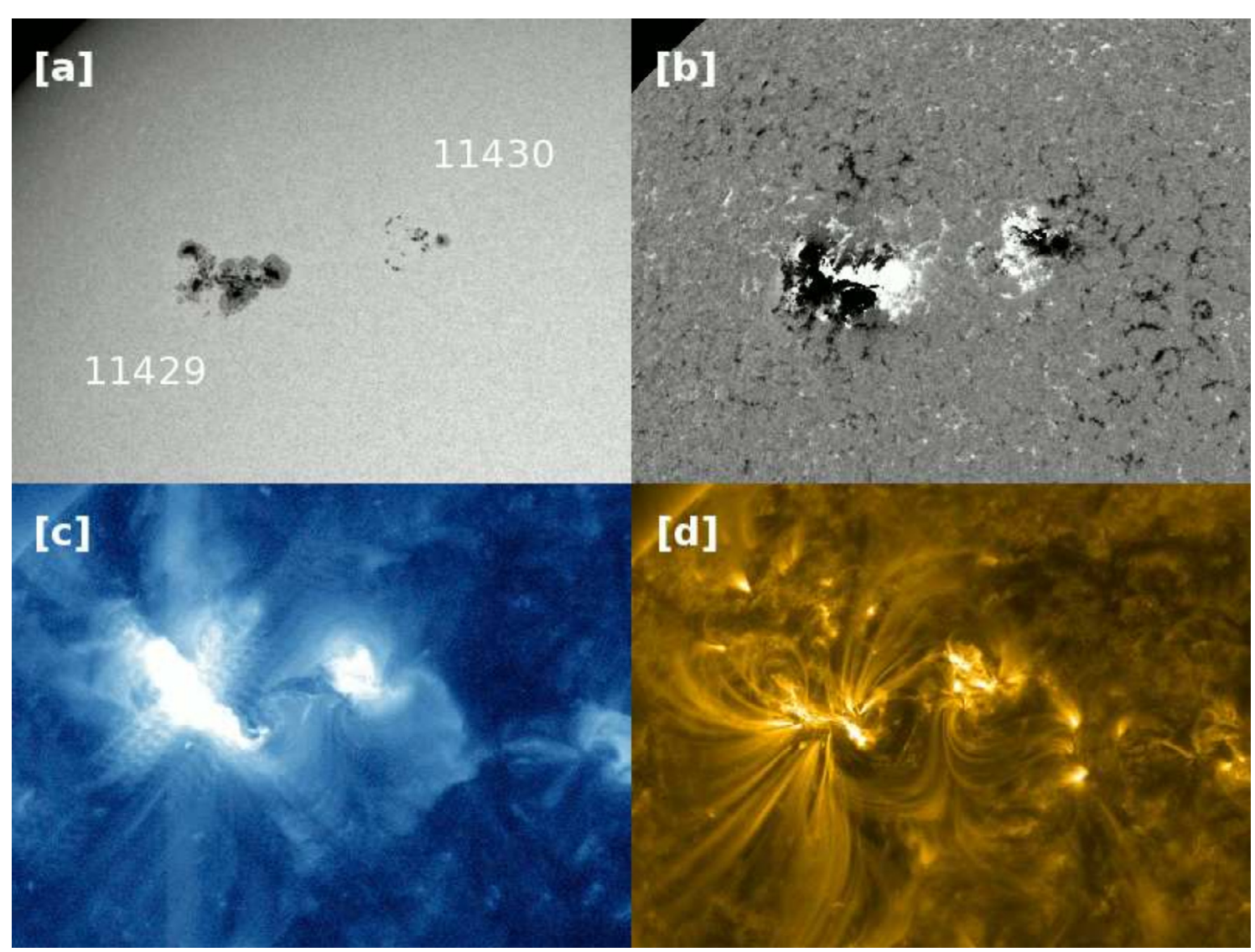

Fig. 1. Active regions AR11429 and AR11430 as observed by SDO imagers around the March 7th event: (a) SDO/HMI Continuum; (b) SDO/ HMI Magnetogram; (c) SDO/AIA $335 \AA$; [d] SDO/AIA $171 \AA$.

longitudinal positions that are favourable for direct transport of SEPs in circumterrestrial space along interplanetary magnetic field lines. Empirical knowledge (Benz 2008) tells us that intense flares are less likely than faint ones. Effectively, power-law slopes are reported for flare peak fluxes, which are invariant through various solar cycles, both using observations and numerical models (e.g. Viticchié et al. 2006; Aschwanden \& Freeland 2012).

In order to quantify the frequency of severe solar flares extensive surveys of stars of Sun-like stars have been performed and indirect arguments, for example based on records of sunspots, have been adopted (Schrijver et al. 2012). A reasonable estimate is that there about a $10 \%$ chance of a flare larger than about X30 in the next 30 years.

Currently, the observed March 7th, 2012 flare (X5.4) has been the second largest event of cycle 24 after the flare that occurred on August 9th, 2011 (X6.9). The flare occurred in the sunspot region AR11429 and produced an intense Earthward CME which reportedly could have temporarily knocked some American military satellites offline ${ }^{2}$ and heated the Earth's upper atmosphere with the largest dose of infrared radiation since 2005. During the event, from March 8th to March 10th, the thermosphere absorbed about 26 billion $\mathrm{kW}$ h of energy ${ }^{3}$.

The particle flux associated with the event was also detected on board the International Space Station (ISS) by the ALTEA (Anomalous Long Term Effects on Astronauts) active particle detector. This instrument was expressly designed to measure the effect of the exposure of crew members to cosmic radiation.

\footnotetext{
${ }^{2}$ US News archives on 2012/03/22 by Koebler J.

${ }^{3}$ NASA Science News archives on 2012/03/22 by Phillips T.
}

It integrates several diagnostic technologies and permits the investigation of the impacts that the particle flux has on the human central nervous system functions (Narici 2008) and therefore provides an assessment of the radiation environment in the ISS (Di Fino et al. 2011; Larosa et al. 2011). This task is accomplished by its active particle detector composed by silicon telescopes (either 3 or 6). The detector is able to achieve real-time measurements of the radiation environment with nuclear discrimination (Zaconte et al. 2008, 2010a, 2010b; Di Fino et al. 2012).

On board the ISS since July 2006, the ALTEA telescope was operative between August 2006 and November 2012 in different experimental setups (i.e. configuration, position and orientation) inside the US Lab and Columbus modules of the ISS. The total effective observation time has been more than three years.

In this paper we present the first detailed analysis of the ALTEA data related to the March 7th, 2012 flare (X5.4) and the associated CME.

\section{Solar activity. The March 7, 2012 event}

The Sun was particularly active in the first half of 2012 . Various active regions (AR) produced a significant number of flares and Coronal Mass Ejections (CMEs). The source of the event of March 7th was AR111429 (see Fig. 1). Elmhamdi et al. (2013) studied the evolution of AR11429, especially in relation to the flares in early March; Joshi et al. (2013) studied SEP events caused by a CME on January 23; Hao et al. (2012) studied a white light flare 
L. Di Fino et al.: The March 7th, 2012 X5.4 flare detected by ALTEA on board the ISS

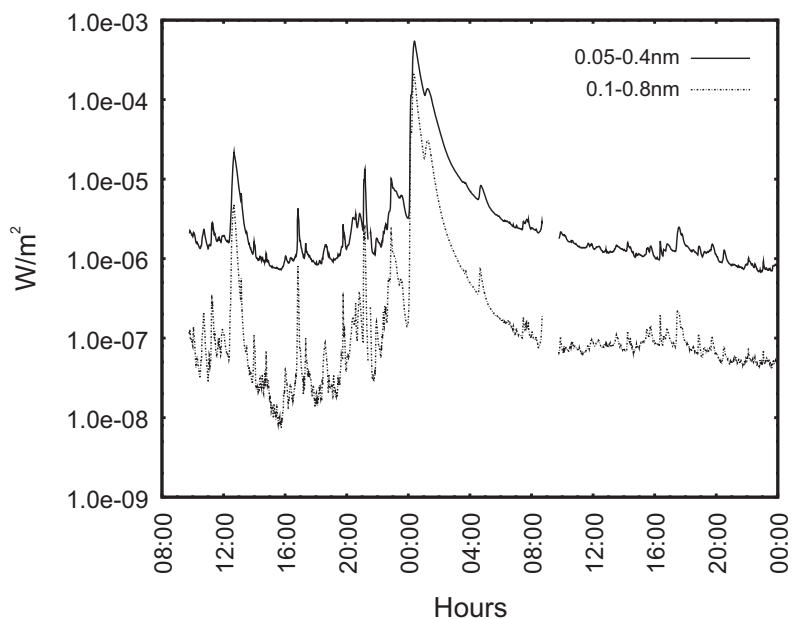

Fig. 2. GOES 15 X-Ray Flux measurement from 08:00 March 6th to 00:00 March 8th, 2012.

occurring on March 9th; Struminsky (2013) studied proton events of January 27th and May 17th.

At the time of the flare emission, AR11429 was located around N17E30 on the Sun. It was composed of several connected sunspots (Fig. 1a). From the magnetic point of view (Fig. 1b), its classification according to the Hale system was $\beta \gamma \delta / \beta \delta$. We recall that a $\beta \gamma$ region is a sunspot group that is bipolar but which is sufficiently complex that no single, continuous line can be drawn between spots of opposite polarities. The $\delta$ qualifier indicates that there are umbrae separated by less than $2^{\circ}$ within one single penumbra that have opposite polarity.

The classification according to the McIntosh (modified Zurich) system was Dkc/Dkc: the $D$ indicates a bipolar sunspot group with penumbra on both ends of the group, whose longitudinal extent does not exceeds $10^{\circ}$; the $k$ indicates that the group is large and asymmetric; the $c$ indicates the group is compact, with many spots between leading and following ends and at least one of these with a full penumbra. Full explanation of the classification systems can be found, for example, on the Solar Influences Data Analysis Center of the Royal Observatory of Belgium website (http://sidc.oma.be/educational/ classification.php).

Regardless of the classification system used, the group was characterized by great magnetic complexity, normally an indicator of high flaring probability. Moreover, the extreme-UV AIA image of AR11429 (Fig. 1c,d) confirms the complexity of this region and highlights its connection with the nearby region AR11430, the latter however not being the origin of any noticeable contemporary events.

AR11429 flared repeatedly between the 5th and the 7th of March 2012. Of these flares, two were X-class events. The first, measured by the GOES satellites as an X1.1 occurred at 02:30 UT on the 5th. It produced a full-halo CME whose speeds where measured at $\simeq 1300 \mathrm{~km} \mathrm{~s}^{-1}$. The second flare, which is the subject of the present work and was classified as X5.4 (Fig. 1c), occurred around 00:15 UT on the 7th. Also this flare was accompanied by a full-halo CME, whose speed was recorded at $\simeq 2300 \mathrm{~km} \mathrm{~s}^{-1}$ (Elmhamdi et al. 2013).

As a matter of fact, the X5.4 flare was a fast sequence of distinct events. The X-ray flux (again measured by the GOES satellites and reported in Fig. 2) shows at least two distinct

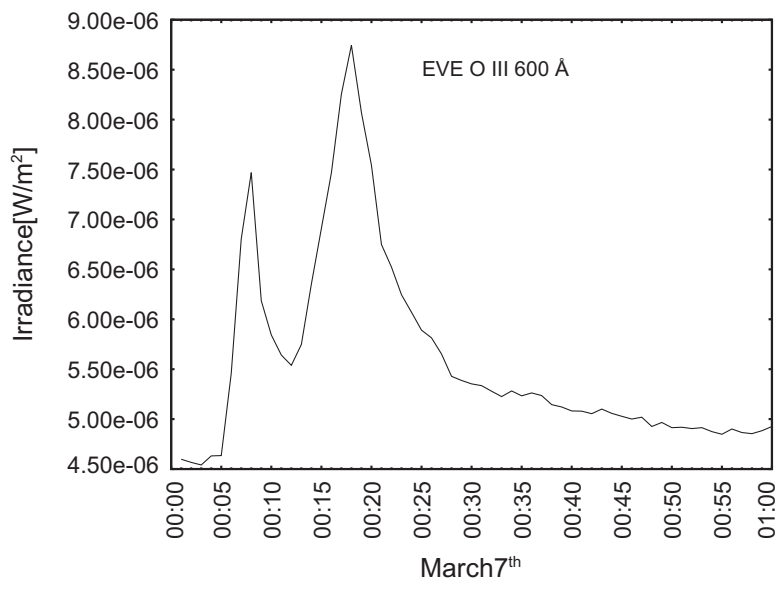

Fig. 3. Irradiance time series of the extreme-UV OIII line at $600 \AA$, recorded by SDO/EVE from 00:00 to 01:00 March 7th, 2012.

peaks between 00:02 UT to 00:24 UT. Emission lines of the solar corona spectrum, as observed by the high temporal cadence extreme-UV detectors of the SDO/EVE instrument, better unveil this double event: in Figure 3 the two sub-events are fully resolved in the case of O III line at $60 \mathrm{~nm}$ (see Del Zanna \& Woods 2013).

From the point of view of the particle emissions related to such event, the CME associated with the R3 (Strong) radio blackout event arrived at ACE (i.e. at Lagrangian point 1) at 10:45 UT March 8th. Closer to the Earth, the proton flux measured by the GOES satellites increased starting from March 7th and the peak of the component over $10 \mathrm{MeV}$ was recorded at 6530 particle $\mathrm{s}^{-1} \mathrm{~cm}^{-2} \mathrm{sr}^{-1}$ around 12:00 of March 8th; the peak of the most energetic proton component (over $100 \mathrm{MeV}$ ) was recorded at 6 particle $\mathrm{s}^{-1} \mathrm{~cm}^{-2} \mathrm{sr}^{-1}$ around 15:00 of March 7th. At ground level, the neutron monitor network (data from NMDB: real-time database for high resolution neutron monitor measurements www.nmdb.eu) detected a persistent decrease of the cosmic ray flux (Fig. 4) caused by the Forbush effect (Forbush 1954; Belov et al. 2001).

While passing ACE, the CME magnetic field was measured. Despite its strength, its orientation was not suitable to cause strong geomagnetic storms at that moment. But on March 9th, the magnetic field orientation changed engendering a geomagnetic storm which was recorded at Earth with $K p$ measured between 5 and 6 .

\section{Instrument and method}

The ALTEA space particle detection system, called SDS (Silicon Detector System), includes six identical particle telescopes (SDU, Silicon Detector Units) arranged on an adaptable mounting system. Each SDU is able to determine the energy loss and the trajectory of the penetrating cosmic rays ${ }^{4}$. A single SDU consists of three pairs of silicon ladders, each ladder composed of two silicon chips segmented into 32 strips with $2.5 \mathrm{~mm}$ pitch. Strip segmentation on each ladder is alternatively oriented along orthogonal directions in order to use the two strip coordinates and the height of the ladder pair into the detector as three coordinates in space. Each silicon chip has a size of

\footnotetext{
${ }^{4}$ without discrimination between forward and backward moving particles.
} 


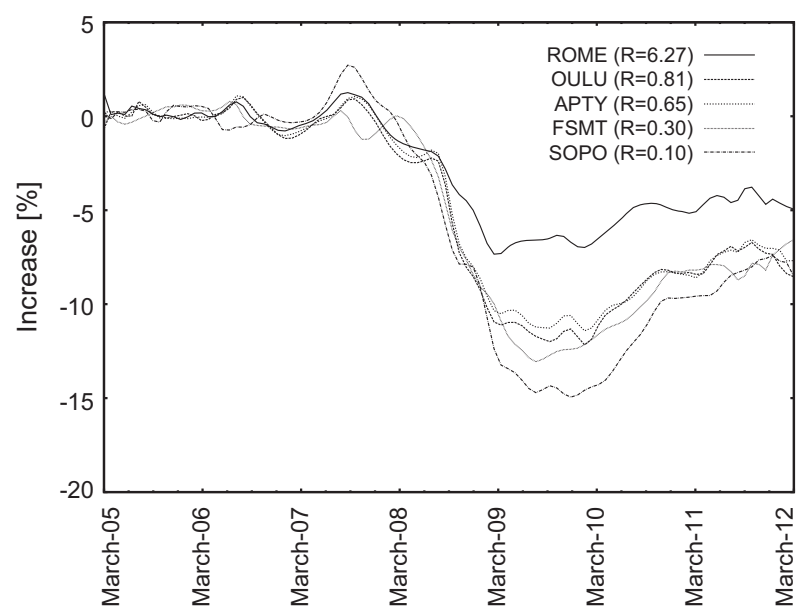

Fig. 4. Time profiles of the cosmic-ray intensity during the March 7 th event, as it was recorded by a number of neutron monitors with different rigidity: Rome, Oulu, Apatity (APTY), Fort Smith (FSMT), and South Pole (SOPO).

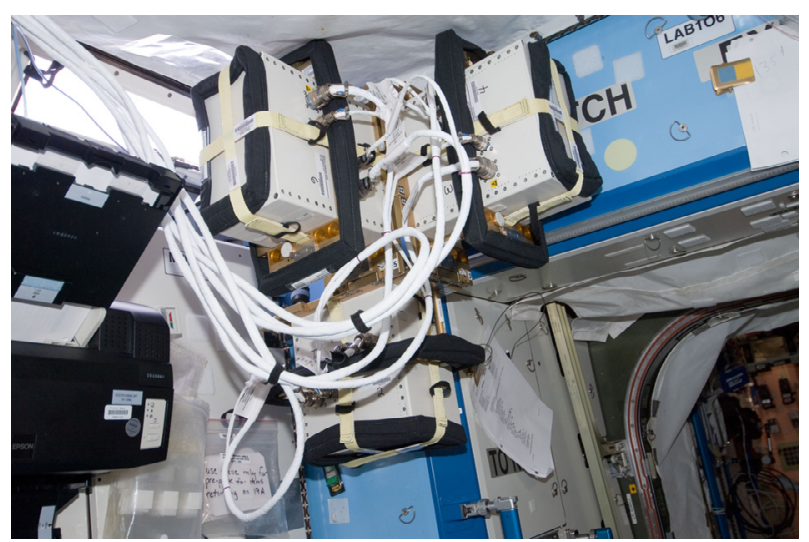

Fig. 5. The ALTEA experiment in Shield-Survey 3D configuration in the USLab module on board the ISS.

$8 \mathrm{~cm} \times 8 \mathrm{~cm}$, and a thickness of $380 \mu \mathrm{m}$. The geometrical factor of a single SDU is $230 \mathrm{~cm}^{2}$ sr. The energy resolution of the detector ranges from a threshold of $2.9 \pm 0.2{\mathrm{keV} \mu \mathrm{m}^{-1}}^{-1}$

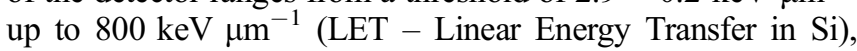
corresponding to ions between $\mathrm{Li}$ and $\mathrm{Fe}$ with a small energy interval for protons and He. The detector is triggered by passthrough particles that release more than the threshold energy on all the odd planes of an SDU. Further details are reported in Zaconte et al. (2008, 2010a). The orbital information provided by the ISS ancillary data allows us to study the particle flux into the three main geomagnetic zones (poles, equator and South Atlantic Anomaly).

From September 2010 to June 2012 ALTEA SDUs were arranged in the ALTEA-Shield/Survey 3D configuration as part of an ESA sponsored experiment. In this setup the SDUs were coupled to form three silicon telescopes with twelve planes; each telescope is pointing in a different $X-Y-Z$ space direction (see Fig. 5).

In this paper we will focus on data acquired from March 6th to March 19th, 2012.

\section{Results and discussion}

Since SDU1 is the silicon telescope which recorded the highest flux during the March 7th solar event, likely because of a combination of low shielding position and solar particle directionality, all the plots shown in the following analysis refer to SDU1. Also, we define the period between February 15th and February 21th, 2012 as reference for the particle flux during "quiet Sun" conditions (indicated as PRE in the plots of Figs. 9 and 11). During this period the activity of the Sun varied from Very Low to Low and no major solar flare or CME were detected.

The signature of the occurrence of a major solar event is evident in the flux recorded by ALTEA: solar events cause flux enhancements much larger than the typical North Pole-EquatorSouth Pole oscillations. In Figure 6 the particle flux integrated over one minute shows a flux increase (in the polar regions up to tenfold with respect to the quiet Sun period used as reference) during the passages near the magnetic poles from March 7th to March 9th, 2012. The reason why the flux increases only in the second half of the day is probably that exclusively in these periods the geomagnetic cut-off is low enough. This effect is even more evident when plotting the integrated particle flux measured at high latitude (Lat $>45^{\circ}$ or Lat $<-45^{\circ}$ ) versus longitude, as shown in Figure 7. The flux enhancement due to the SPE is visible only at longitudes between $-120^{\circ}$ and $-10^{\circ}$ (left panel of Fig. 7) in the North hemisphere and between $30^{\circ}$ and $-150^{\circ}$ in the South hemisphere (right panel of Fig. 7).

Figure 8 shows the daily flux recorded by the SDU1 of ALTEA between March 6th and March 19th; the flux on 7-8 March is up to 2.5 times the flux of the previous period of quiet solar conditions, while after March 10th the particle flux decreases again at the "quiet Sun" reference level.

The ALTEA capability of measuring the particle energy loss allows us to characterize the incoming flux, assessing which cosmic ray component is affected by the solar event. The energy loss spectra acquired during the most active days of the SPE (7-8 March) are shown (Fig. 9) in comparison with the spectra taken as reference during the quiet Sun period (PRE). The spectra shown in the upper panel of Figure 9 are integrated over the whole orbit, those in the lower panel are integrated only during the time spent in high geomagnetic latitude passages. All the spectra are normalized by the effective acquisition time of the detector, by the geometrical factor and by the binning width of the plot.

In the spectra obtained integrating over the whole orbit, peaks corresponding to relativistic ions between Boron $\left(\simeq 7 \mathrm{KeV} \mu \mathrm{m}^{-1}\right)$ and Oxygen $\left(\simeq 24 \mathrm{KeV} \mu \mathrm{m}^{-1}\right)$ are well distinguishable. Protons and Helium are detectable by ALTEA only in specific kinetic energy ranges, $25-45 \mathrm{MeV}$ for protons and 25-250 MeV/amu for Helium ions (Zaconte et al. 2008, 2010a). During the SPE (7-8 March), the flux increases $\sim 2.5$ times the reference level for LET $<10 \mathrm{keV} \mu \mathrm{m}^{-1}$ due to the proton component, while the $30 \%$ increase for LET up

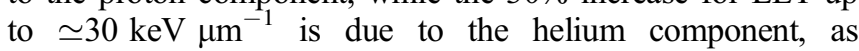
confirmed by SRIM (Stopping and Range of Ions in Matter - http://www.srim.org, see e.g. Ziegler et al. 2010) simulations 5 . Beyond these last LET values, the statistic fails.

\footnotetext{
$\overline{{ }^{5} \text { Note that } 10 \mathrm{keV} \mathrm{\mu m}^{-1}}$ and $35 \mathrm{keV} \mathrm{\mu m}^{-1}$ are, respectively, the maximum energy loss for protons and Helium ions that can be detected by ALTEA detectors.
} 
L. Di Fino et al.: The March 7th, 2012 X5.4 flare detected by ALTEA on board the ISS

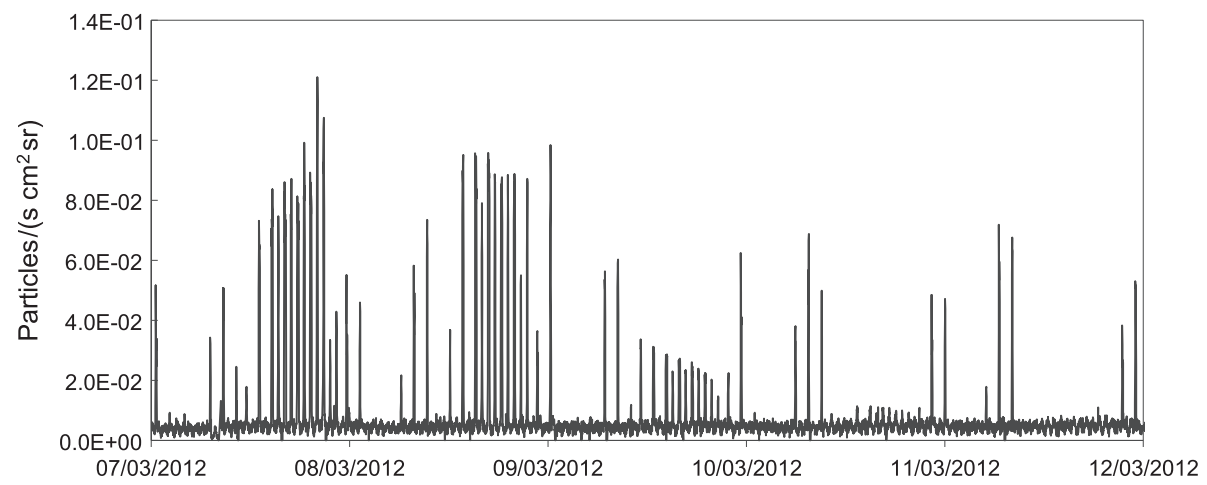

Fig. 6. Particle flux integrated over one minute: the flux enhancement between March 7 th and 9th is the signature of a solar event.
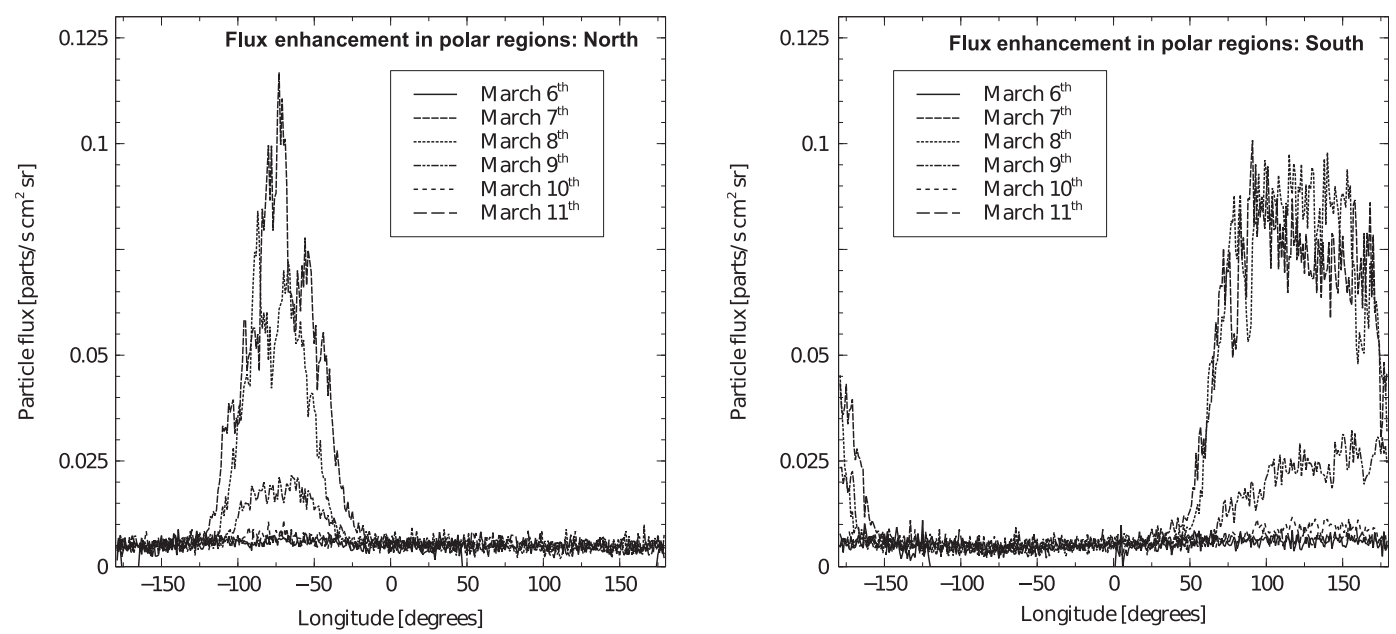

Fig. 7. Flux enhancement in polar regions before, during, and after the SPE. Left panel: the northern hemisphere; right panel: southern hemisphere.

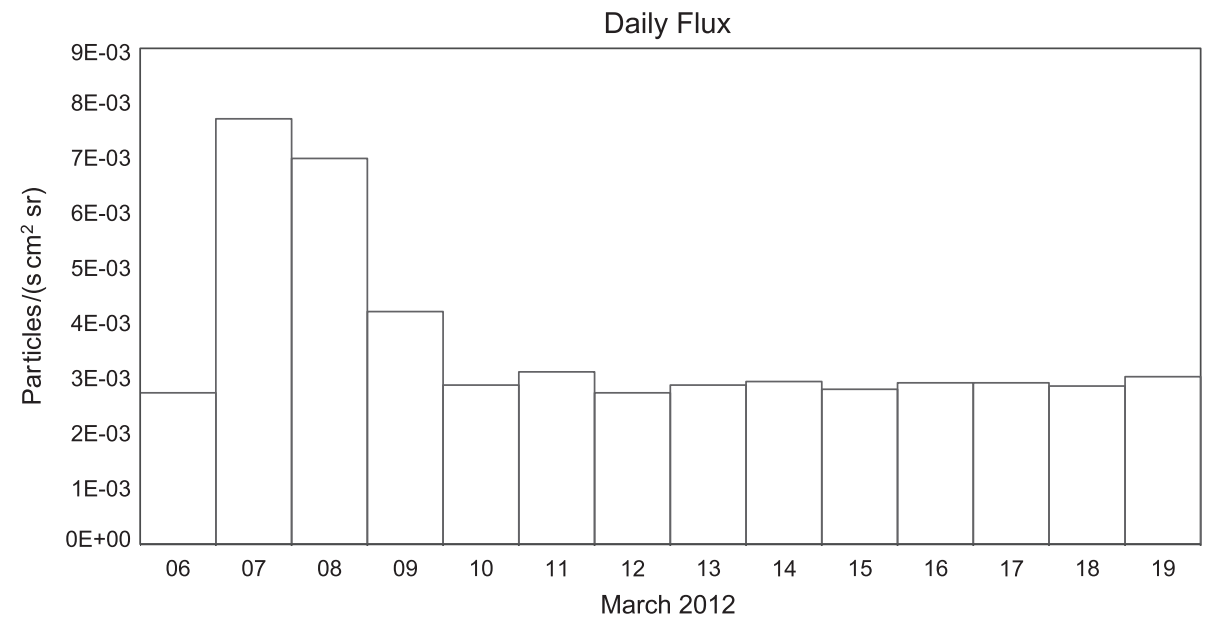

Fig. 8. Daily flux rate recorded by ALTEA SDU1 between March 6th and March 19th, 2012.

As already stated, the spectra reported in the lower panel of Figure 9 are integrated only during the time spent in high geomagnetic latitude passages, i.e. for Mcllwain coordinate $L>3$ (Mcllwain 1961). We recall that, given a point in space and the magnetic field line passing through it, $L$ is the distance (expressed in Earth radii) between the Earth center and the intersection between that field line and the magnetic equatorial plane. Therefore, the spectra integrated on $L>3$ select the 

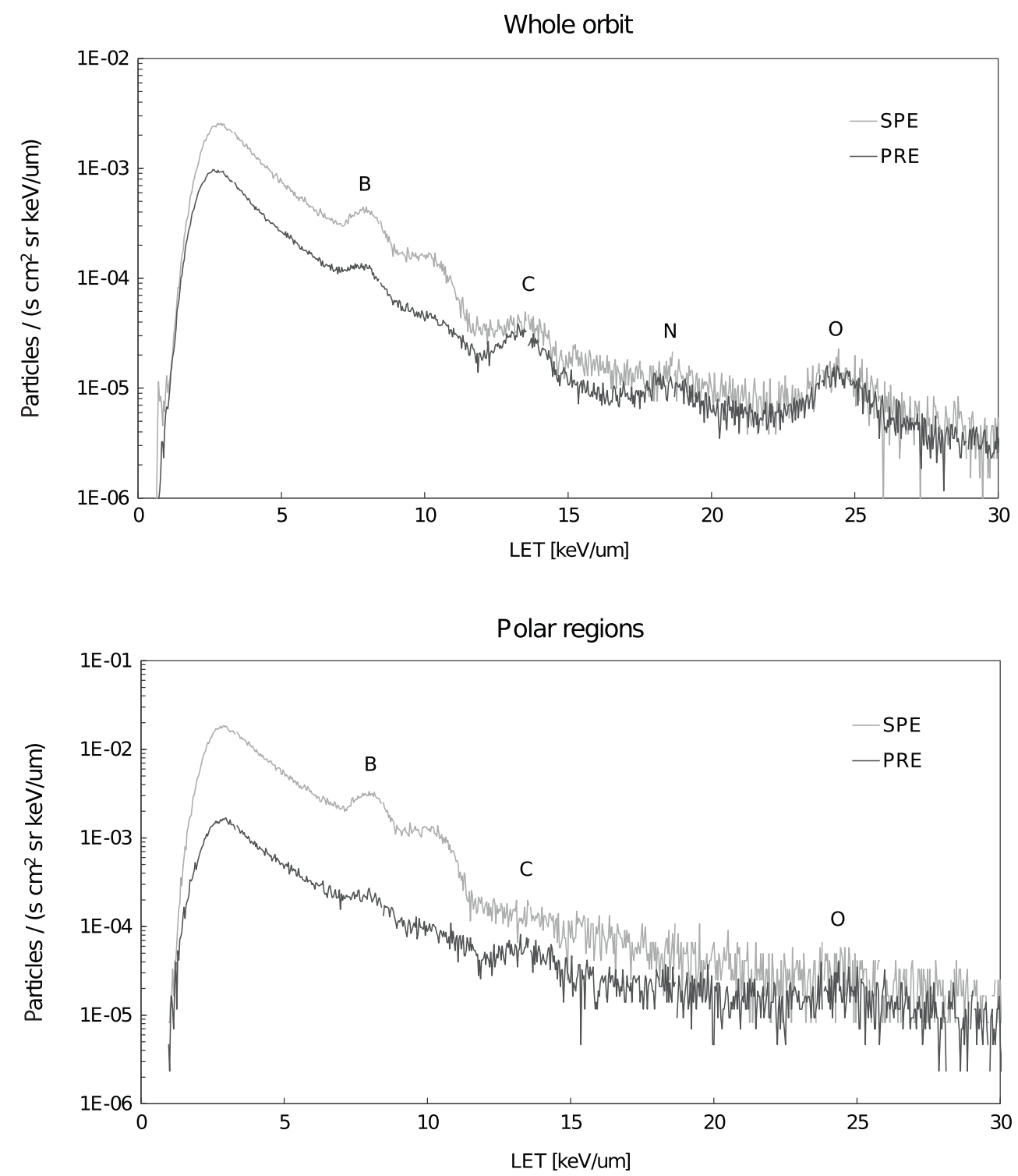

Fig. 9. The SPE energy spectrum compared with the quiet Sun spectrum (PRE). Upper panel: energy loss spectra integrated over the whole orbit. Lower panel: energy loss spectra integrated in the polar regions only.

particles penetrating the polar regions, where the energy cutoff is lower with respect to the equatorial region. As already seen in Figure 7, the SPE particles can penetrate the geomagnetic shield only nearby the magnetic poles. The plots reported in the lower panel of Figure 9 show that the spectrum increase in the polar region is about one order of magnitude for LET $<10 \mathrm{keV} \mu \mathrm{m}^{-1}$, confirming that the SPE flux enhancement is caused mainly by protons and Helium nuclei.

In order to assess the radiation risk correlated to SPEs, the daily dose rate is presented in Figure 10. On March 7 the dose rate is almost double the typical dose rate in standard conditions. As already stated, the SPE effect is much more evident at high latitudes ( $L>3$ polar regions). It is therefore interesting to compare the dose and dose equivalent variations, on the whole orbit and in the $L>3$ polar regions, the latter being more representative of an outer space environment.

To calculate dose and dose equivalent, first we transform the LET in Si into the LET in water according to the following formula from Benton et al. (2010):

$$
\log \left(\operatorname{LET}_{\mathrm{H}_{2} \mathrm{O}}\right)=-0.2902+1.025 \log \left(\mathrm{LET}_{\mathrm{Si}}\right) .
$$

The differential absorbed dose rate (in $[\mathrm{Gy} / \mathrm{s}]$ ) is then obtained from:

$$
\text { Dose }=4 \pi \times 1.6 \times 10^{-9} \mathrm{LET}_{\mathrm{H}_{2} \mathrm{O}},
$$

where the $\mathrm{LET}_{\mathrm{H}_{2} \mathrm{O}}$ rate is measured in $\left[\mathrm{keV} \mu \mathrm{m}^{-1}\right.$ $\mathrm{s}^{-1} \mathrm{~cm}^{-2} \mathrm{sr}^{-1}$ ] (Benton \& Benton 2001). The dose equivalent is calculated as $Q \times$ Dose, where the quality factor $Q$ is defined as per ICRP recommendations (ICRP 1991). The dose equivalent rate is measured in $\left[\mathrm{Sv} \mathrm{s}{ }^{-1}\right]$.

Figure 11 shows the integrated dose and dose equivalent on the whole orbit and in the polar regions only. Although in a limited acceptance window for protons, the integrated dose measured by ALTEA during this SPE increases up to twice the reference values in the whole orbit and more than five times in the polar regions. The dose equivalent takes into 
L. Di Fino et al.: The March 7th, 2012 X5.4 flare detected by ALTEA on board the ISS

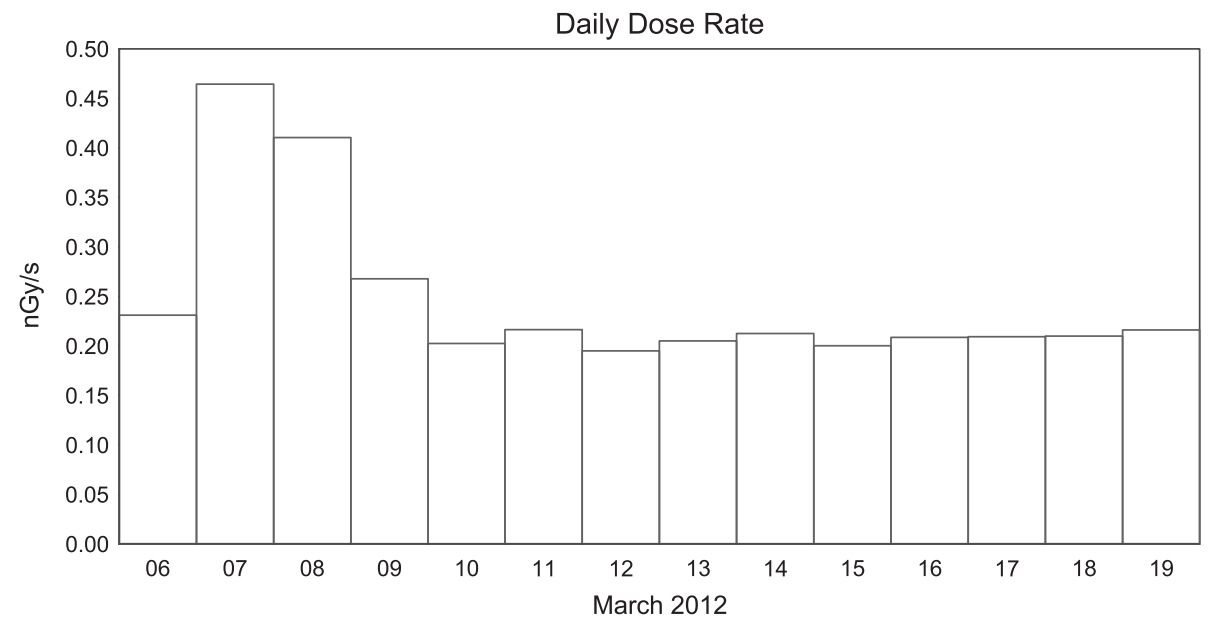

Fig. 10. Daily dose rate recorded by ALTEA SDU1 between March 6th and March 19th, 2012.
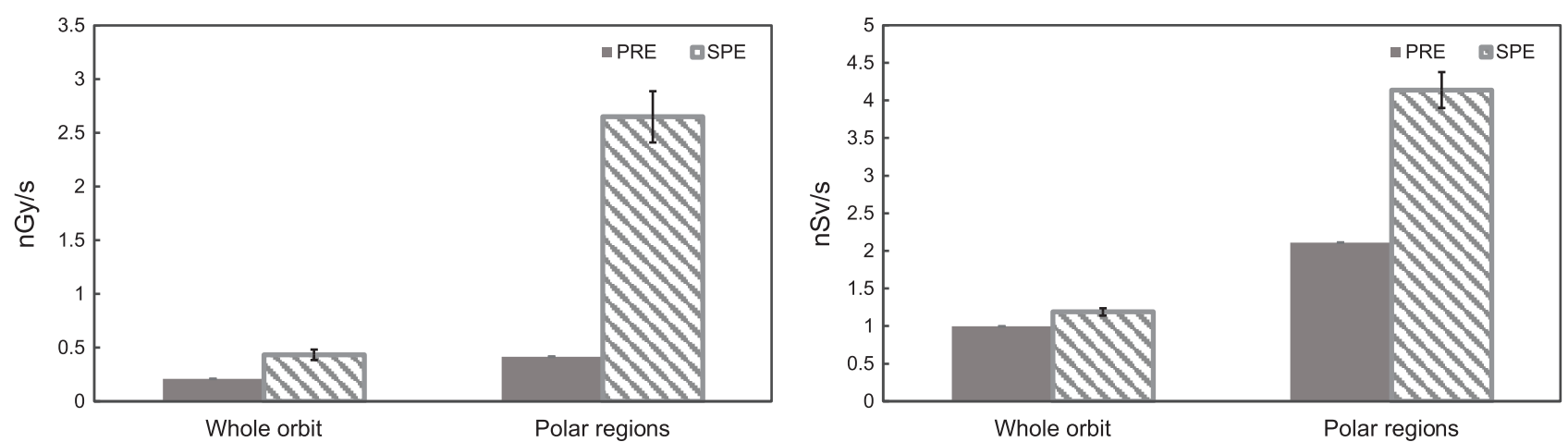

Fig. 11. Time averaged dose (left panel) and dose equivalent (right panel) during the SPE and during the quiet Sun period (PRE). The error bars represent statistical errors.

account the ability of each ion to produce biological damage (higher for higher deposited energy). The contribution to dose equivalent of protons and light ions is therefore very low. This minimizes the effect on dose equivalent of the enhanced flux of protons and light ions due to the SPE and makes the dose equivalent rate have almost no variations in the whole orbit and increase less than twofold in polar regions.

\section{Conclusions}

In this work we presented the charged radiation flux measured by ALTEA on board the ISS during the March 7th, 2012 solar particle event. The ALTEA experiment was in its ALTEAShield/Survey 3D configuration and was able to detect the incoming flux of SEPs generated by the solar event.

- As seen by ALTEA, during the SPE the flux is enhanced up to 10 times with respect to "quiet Sun" conditions.

- The energy loss spectra show that protons and Helium components are affected the most by the SPE.

- The comparison of the spectra integrated during high geo-magnetic latitude passages $(L>3)$ of the ISS, allowed ALTEA to estimate the efficiency of SEP penetration at the polar regions and the consequent flux enhancement.
- Those flux variations result in a dose and dose equivalent increase, which are particularly strong $(5 \times$ and $2 \times$, respectively) at high geomagnetic latitudes.

In addition to introducing ALTEA as a prospective Space Weather monitor, these results are of specific relevance for charged radiation risk assessment in space habitats during a SPE.

Acknowledgements. ALTEA has been funded by the Italian Space Agency (ASI) and the Italian National Institute for Nuclear Physics (INFN).

The ALTEA program acknowledges support from ESA (experiment ALTEA_shield) both on the ISS and on ground, NASA and the ISS crews for their support in orbit. The ISS is an international partnership with contributions from the United States, Russia, Japan and Canada, Europe.

R.P., S.S. and M.L. acknowledge support by the SPARC: Space Awareness for Critical Infrastructure project. SPARC has been financed by the Prevention, Preparedness and Consequence Management of Terrorism and other Security-related Risks Programme European Commission - Directorate-General Home Affairs under the grant number: HOME/2011/CIPS/AG/4000002119.

Some images are courtesy of NASA/SDO and the AIA, EVE, and HMI science teams.

We acknowledge the NMDB database (www.nmdb.eu), founded under the European Union's FP7 programme (Contract no. 213007) for providing data. Data used was acquired at Rome, Oulu, Apatity, Fort Smith, and South Pole (SOPO) stations.

We acknowledge the NOAA-NASA GOES Project for providing data. 


\section{References}

Aschwanden, M.J., and S.L. Freeland, Automated solar flare statistics in soft X-rays over 37 years of GOES observations: the invariance of self-organized criticality during three solar cycles, Astrophys. J., 754, 112, DOI: 10.1088/0004-637X/754/2/112, 2012.

Belov, A.V., E.A. Eroshenko, V.A. Oleneva, A.B. Struminsky, and V.G. Yanke, What determines the magnitude of forbush decreases? Adv. Space Res., 27, 625, DOI: 10.1016/S0273-1177(01)00095-3, 2001.

Benton, E.R., and E.V. Benton, Space radiation dosimetry in lowEarth orbit and beyond, Nucl. Instrum. Methods Phys. Res. B, 184, 255, DOI: 10.1016/S0168-583X(01)00748-0, 2001.

Benton, E.R., E.V. Benton, and A.L. Frank, Conversion between different forms of LET, Radiat. Meas., 45 (8), 957, DOI: 10.1016/j.radmeas.2010.05.008, 2010.

Benz, A.O., Flare Observations, Living Rev. Sol. Phys., 5, 1, http://www.livingreviews.org/lrsp-2008-1 [cit. 2013-12-23] DOI: $10.12942 / \mathrm{lrsp}-2008-1,2008$

Del Zanna, G., and T.N. Woods, Spectral diagnostics with the SDO EVE flare lines, $A \& A, \mathbf{5 5 5}, \mathrm{A} 59$, DOI: 10.1051/0004-6361/201220988, 2013.

Di Fino, L., M. Casolino, C. De Santis, M. Larosa, C. La Tessa, L. Narici, P. Picozza, and V. Zaconte, Heavy ions anisotropy measured by ALTEA in the International Space Station, Radiat. Res., 176, 397, DOI: 10.1667/RR2179.1, 2011.

Di Fino, L., V. Zaconte, A. Ciccotelli, M. Larosa, and L. Narici, Fast Probabilistic Particle Identification algorithm using silicon strip detectors, Adv. Space Res., 50, 408,

DOI: 10.1016/j.asr.2012.04.015, 2012.

Durante, M., and F.A. Cucinotta, Physical basis of radiation protection in space travel, Rev. Mod. Phys., 83, 1245, DOI: 10.1103/RevModPhys.83.1245, 2011.

Elmhamdi, A., A.S. Kordi, H.A. Al-Trabulsy, M. El-Nawawy, A.A. Ibrahim, N. Ben Nessib, M.A. Abdel-Sabour, and Z.A. Al-Mostafa, Observations and analysis of NOAA AR 11429 at KSU-Astronomical Observatory, New Astron., 23, 73, DOI: 10.1016/j.newast.2013.03.007, 2013.

Forbush, S.E., World-wide cosmic-ray variations, J. Geophys. Res., 59, 525, DOI: 10.1029/JZ059i004p00525, 1954.

Hao, Q., Y. Guo, Y. Dai, M.D. Ding, Z. Li, X.Y. Zhang, and C. Fang, Understanding the white-light flare on 2012 March 9: evidence of a two-step magnetic reconnection, $A \& A, \mathbf{5 4 4}, \mathrm{L} 17$, DOI: 10.1051/0004-6361/201219941, 2012.

ICRP, 1990 Recommendations of the International Commission on Radiological Protection. ICRP Publication 60, Ann. ICRP, 21 (1-3), 1991.

Joshi, N.C., W. Uddin, A.K. Srivastava, R. Chandra, R.N. Gopalswamy, et al., A multiwavelength study of eruptive events on January 23, 2012 associated with a major solar energetic particle event, $A d v$. Space Res., 52, 1, DOI: 10.1016/j.asr.2013.03.009, 2013.

Lapenta, G., V. Pierrard, R. Keppens, S. Markidis, and S. Poedts, SWIFF: space weather integrated forecasting framework, J. Space Weather Space Clim., 3, A05, DOI: 10.1051/swsc/2013027, 2013.

Larosa, M., F. Agostini, M. Casolino, C. De Santis, L. Di Fino, C. La Tessa, L. Narici, P. Picozza, A. Rinaldi, and V. Zaconte, Ion rates in the International Space Station during the December 2006 Solar Particle Event, J. Phys. G: Nucl. Phys., 38, 095102, DOI: 10.1088/0954-3899/38/9/095102, 2011.

McIlwain, C.E., Coordinates for mapping the distribution of magnetically trapped particles, J. Geophys. Res., 66, 3681, DOI: 10.1029/JZ066i011p03681, 1961.

Narici, L., Heavy ions light flashes and brain functions: recent observations at accelerators and in spaceflight, New J. Phys., 10, 075010, DOI: 10.1088/1367-2630/10/7/075010, 2008.

Potgieter, M.S., Solar modulation of cosmic rays, Living Rev. Sol. Phys., 10, 3, http://www.livingreviews.org/lrsp-2013-3 [cit. 2013-12-23] DOI: 10.12942/lrsp-2013-3, 2013.

Schimmerling, W., F.A. Cucinotta, and J.W. Wilson, Radiation risk and human space exploration, Adv. Space Res., 31, 27, DOI: 10.1016/S0273-1177(02)00653-1, 2003.

Schrijver, J.C., J. Beer, U. Baltensperger, E.W. Cliver, M. Gudel, H.S. Hudson, K.G. McCracken, R.A. Osten, T. Peter, D.R. Soderblom, I.G. Usoskin, and E.W. Wolff, Estimating the frequency of extremely energetic solar events, based on solar, stellar, lunar, and terrestrial records, J. Geophys. Res.: Space Phys., 117, A8, DOI: 10.1029/2012JA017706, 2012.

Shibata, K., and T. Magara, Solar flares: magnetohydrodynamic processes, Living Rev. Sol. Phys., 8, 6, http://www.livingreviews. org/lrsp-2011-6 [cit. 2013-12-23] DOI: 10.12942/lrsp-2011-6, 2011.

Struminsky, A., Large SEP events of 2012: proton onset and source function, J. Phys: Conf. Ser., 409, 012148, DOI: 10.1088/1742-6596/409/1/012148, 2013.

Tsagouri, I., A. Belehaki, N. Bergeot, C. Cid, V. Delouille, et al., Progress in space weather modeling in an operational environment, J. Space Weather Space Clim., 3, A17,

DOI: 10.1051/swsc/2013037, 2013.

Turner, R., Editor. Foundations of solar particle event risk management strategies. In : Findings of the Risk Management Workshop for Solar Particle Events, publication NASA Grant NAGW-4166, 1996.

Viticchié, B., D. Del Moro, and F. Berrilli, Statistical properties of synthetic nanoflares, Astrophys. J., 652, 1734, DOI: $10.1086 / 508332,2006$

Zaconte, V., F. Belli, V. Bidoli, M. Casolino, L. Di Fino, et al., ALTEA: the instrument calibration, Nucl. Instrum. Methods Phys. Res.: Sect. B, 266, 2070, DOI: 10.1016/j.nimb.2008.02.072, 2008.

Zaconte, V., M. Casolino, L. Di Fino, C. La Tessa, M. Larosa, L. Narici, and P. Picozza, High energy radiation fluences in the ISSUSLab: ion discrimination and particle abundances, Radiat. Meas., 45, 168, DOI: 10.1016/j.radmeas.2010.01.020, 2010a.

Zaconte, V., M. Casolino, C. De Santis, L. Di Fino, C. La Tessa, M. Larosa, L. Narici, and P. Picozza, The radiation environment in the ISS-USLab measured by ALTEA: spectra and relative nuclear abundances in the polar, equatorial and SAA regions, $A d v$. Space Res., 46, 797, DOI: 10.1016/j.asr.2010.02.032, 2010b.

Ziegler, J.F., M.D. Ziegler, and J.P. Biersack, SRIM - The stopping and range of ions in matter (2010), Nucl. Instrum. Methods Phys. Res.: Sect. B, 268, 1818, DOI: $10.1016 /$ j.nimb.2010.02.091, 2010.

Cite this article as: Di Fino L, Zaconte V, Stangalini M, Sparvoli R, Picozza P, et al.: Solar particle event detected by ALTEA on board the International Space Station. J. Space Weather Space Clim., 2014, 4, A19. 